\title{
Systemic sclerosis and chronic lymphocytic leukaemia
}

\author{
Y. Sidi, R. Fadilah, J. Pinkhas and M. Prokocimer \\ Department of Internal Medicine 'D' and Division of Hematology, Beilinson Medical Center, Petah Tikva, \\ Sackler Faculty of Medicine, Tel Aviv University, Israel
}

\begin{abstract}
Summary: The association between the CREST (calcinosis, Raynaud phenomena, oesophageal hypomotility, sclerodactyly and telangiectasia, variant of systemic sclerosis and chronic lymphocytic leukaemia is described in three patients. The present description raises the possibility that the association of chronic lymphocytic leukaemia and systemic sclerosis is non-random.
\end{abstract}

\section{Introduction}

Patients with B-chronic lymphocytic leukaemia (CLL) may develop features of autoimmunity. Coombs' positive haemolytic anaemia occurs in $15 \%$ to $35 \%$ of patients at some time during the course of the disease. ${ }^{1}$ Autoimmune thrombocytopenia and neutropenia have been reported as well. $^{2,3}$ Several authors have described casual occurrence of various autoimmune diseases during the course of CLL. ${ }^{4,5}$ Nevertheless, the prevalence of non-haematological autoantibodies is not increased when compared to age-matched controls. ${ }^{6,7}$

Recently, we studied three patients with a combination of CLL and systemic sclerosis (SS). This finding raised the possibility that this association may be non-random.

\section{Case reports}

\section{Case 1}

A 61 year old female was admitted for evaluation of shoulder pains and recurrent arthritis of the knee joints. Diagnosis of CREST variant of SS with myositis was established on the basis of repeated episodes of Raynaud phenomena in the last 5 years, multiple telangiectatic lesions on the hands, sclerodactyly and tightness of the facial skin, subcutaneous calcifications and dilated oesophagus with disturbed peristalsis. Electromyography, deltoid muscle biopsy and elevated creatine phosphokinase confirmed the presence of myositis. She had been diagnosed 3 years previously as having CLL, Rai stage 0, in view of persistent lymphocytosis $30 \times 10^{9} / 1,70 \%$ of B type. B phenotype was confirmed by the presence of low intensity

Correspondence: Y. Sidi, M.D., Department of Internal Medicine 'D', Beilinson Medical Center, 49100 Petach Tikva, Israel

Accepted: 31 May 1990 immunoglobulin staining on the leukaemic cells and the presence of receptors for mouse erythrocytes. She was treated for 6 months with prednisone $1 \mathrm{mg} / \mathrm{kg}$. Following this therapy no improvement was noted. However, over the next 2 years her clinical condition remained stable without further treatment.

\section{Case 2}

A 77 year old male was admitted for evaluation of headache and recent mental deterioration. A clinical diagnosis of right parietal lobe meningioma was done by computerized tomography of the skull. The patient refused surgery. Diagnosis of CREST variant of SS was established on the basis of 20 years' history of Raynaud phenomena leading to necrotic digital lesions, decreased motility of the oesophagus, and wide atonic duodenum and jejunal loops, sclerodactyly, multiple palmar and chest telangiectatic lesions. CLL, Rai stage 1 was diagnosed at the same time by the findings of generalized lymphadenopathy without hepatosplenomegaly and white cell count of $48 \times 10^{9} / 1$ with $62 \%$ lymphocytes, positive for surface immunoglobulins and receptors for mouse erythrocytes. The patient suffered recurrent events of Gram-negative bacteraemia and diarrhoea, which improved after intensive oral and broad spectrum parenteral antibiotic treatment. Two years later his condition is satisfactory without any deterioration of either the SS and CLL or in his neurological status.

\section{Case 3}

A 47 year old male was admitted for evaluation of recurrent diarrhoea and marked weight loss over the past 6 months. The diagnosis of CREST variant of SS was made 3 years previously on the basis of 11 years' history of Raynaud phenomena, sclerodac- 
tyly, subcutaneous calcifications and multiple palmar and chest telangiectatic lesions. A barium meal revealed oesophageal hypomotility and dilatation of upper jejunal loops. Two years later he suffered repeated arthritis of both knees and hip joints. Lymphadenopathy and splenomegaly were found. CLL, Rai stage 2, was diagnosed by white cell count up to $40 \times 10^{9} / 1$ with $85 \%$ lymphocytes positive for surface immunoglobulins and receptors for mouse erythrocytes. The hospital course was complicated by repeated paralytic ileus and bilateral bronchopneumonia from which he died. Permission for autopsy was not granted.

\section{Discussion}

Various autoimmune phenomena are known to complicate the course of CLL. However, the propensity to develop autoantibodies is believed to be restricted to the haematopoietic system. ${ }^{8}$ The patients may develop haemolytic anaemia, thrombocytopenic purpura or neutropenia related to autoantibodies to red blood cells, platelets or neutrophils, respectively. ${ }^{1-3,8} \mathrm{We}$ and others noticed that the prevalence of autoimmune diseases and non-haematological autoantibodies in a large unselected series of patients with B-CLL was not increased. ${ }^{6,7}$

However, sporadic occurrence of the following autoimmune diseases in B-CLL patients have been recorded: autoimmune hyperthyroidism, ${ }^{9}$ immune deposit nephritis ${ }^{10}$ and cryoglobulinaemia with cold urticaria. ${ }^{11}$ Dameshek and Miller ${ }^{4,5}$ reported coexistence of B-CLL with allergic vasculitis, chronic glomerulonephritis, ulcerative colitis, systemic lupus erythematosus and rheumatoid arthritis. The association of pernicious anaemia, ${ }^{12}$ nephrotic syndrome, ${ }^{13}$ bullous pemphigoid with B-CLL has also been described..$^{14}$

The present study suggests a novel association between SS and B-CLL. We are aware of only two previous reports suggesting the possibility of such association. ${ }^{15,16}$ However, in all of them evaluation of probability of the coincidental combination was not performed. We have performed a computerized survey of a list of diagnoses of $1,223,971$ patients hospitalized in seven general medical centres affiliated with the Kupat Holim Health Organization in Israel between the years 1974-1988. In this preliminary survey the combined diagnosis of CLL and SS appeared in $2.4 \times 10^{-6}$ of the patients. This incidence is 30-fold higher than the expected random coincidence calculated by the multiplication of the incidence of diagnosis of CLL or SS in the same patient population. However, no real epidemiological conclusions can be derived from such a simple survey. It can be biased by the selection of hospitalized population. The exact connection between both diseases has to be determined by a prospective study in large groups of patients suffering from CLL or SS.

\section{References}

1. Bergsagel, D.E. The chronic leukemias: a review of disease manifestations and the aims of therapy. Can Med Assoc $J$ 1967, 96: 1615-1620.

2. Carey, R.W., McGinnis, A., Jakobson, B.M. \& Carralho, A. Idiopathic thrombocytopenic purpura complicating chronic lymphocytic leukemia. Arch Intern Med 1976, 136: 62-66.

3. Rustagi, P., Zolkowski, H.T., Currie, M. \& Luge, G. Anti-granulocyte antibodies in chronic lymphocytic leukemia and other chronic lymphoproliferative disorders. Blood 1983, 62 (Suppl. 1): 106 (Abstract).

4. Dameshek, W. Chronic lymphocytic leukemia - an accumulative disease of immunologically incompetent lymphocytes. Blood 1967, 29: 566-584.

5. Miller, D.G. Patterns of immunological deficiency in lymphomas and leukemias. Ann Intern Med 1962, 57: 703-715.

6. Hamblin, T.J., Oscier, D.G. \& Young, B.J. Autoimmunity in chronic lymphocytic leukemia. J Clin Pathol 1986, 39: 713-716.

7. Lischner, M., Prokocimer, M., Zolberg, A. \& Shaklai, M. Autoimmunity in chronic lymphocytic leukemia. Postgrad Med J 1988, 64: 590-592.

8. Gale, R.P. \& Foon, K.A. Chronic lymphocytic leukemia. Recent advances in biology and treatment. Ann Intern Med 1985, 103: $101-120$.

9. Havybenstock, A. \& Zalasky, R. Autoimmune hyperthyroidism and thrombocytopenia developed in a patient with chronic lymphocytic leukemia. Am J Hematol 1985, 19. 281-283.

10. Gilboa, N., Durante, D., Guggenheim, S. et al. Immune deposit nephritis and single component cryoglobulinemia associated with chronic lymphocytic leukemia. Nephron 1979, 24: 223-231.

11. Rawnsley, H.M. \& Shelley, W.B. Cold urticaria with cryoglobulinemia in a patient with chronic lymphocytic leukemia Arch Dermatol 1986, 98: 12-17.

12. Parker, A.C. \& Bennett, M. Pernicious anemia and lymphoproliferative disease. Scand J Haematol 1976, 17: 395-397.

13. Feehilly, J., Hutchinson, R.M., Masckay, E.G. \& Walls, J. Recurrent proteinuria in chronic lymphocytic leukemia. Clin Nephrol 1981, 16: 51-54.

14. Goodnough, L.T. \& Muri, A. Bullous pemphigoid as a manifestation of chronic lymphocytic leukemia. Arch Intern Med 1980, 140: 1526-1527.

15. Duncan, S.C. \& Winkelmann, R.K. Cancer and scleroderma. Arch Dermatol 1979, 115: 950-955.

16. Doyle, J.A., Connolly, S.M. \& Hoagland, H.C. Hematologic disease in scleroderma. Acta Derm Venereal (Stockh.) 1985, 65: $521-529$. 